\title{
LOWER BOUNDS FOR RELATIVE CLASS NUMBERS OF CM-FIELDS
}

\author{
STÉPHANE LOUBOUTIN
}

(Communicated by William Adams)

\begin{abstract}
Let $\mathbf{K}$ be a CM-field that is a quadratic extension of a totally real number field $\mathbf{k}$. Under a technical assumption, we show that the relative class number of $\mathbf{K}$ is large compared with the absolute value of the discriminant of $\mathbf{K}$, provided that the Dedekind zeta function of $\mathbf{k}$ has a real zero $s$ such that $0<s<1$. This result will enable us to get sharp upper bounds on conductors of totally imaginary abelian number fields with class number one or with prescribed ideal class groups.
\end{abstract}

Let $\mathbf{K}$ be a CM-field that is a quadratic extension of a totally real number field $\mathbf{k}$.

If the Dedekind zeta function of $\mathbf{K}$ is nonpositive at some $s_{0}$ that belongs to the interval ]0, 1[, then it is well known that we can get good lower bounds for the residue at $s=1$ of this zeta function and for the relative class number of $\mathbf{K}$. In Proposition A, we give explicit forms of such a result. They will enable us to consider in Corollary $c$ the class number one problem for cyclotomic fields in a more efficient way than those one can find in the literature (see [7, 12]).

Now, if the Dedekind zeta function of $\mathbf{k}$ has a zero in $] 0,1[$, then in Theorem 1 we give lower bounds for the relative class number of $\mathbf{K}$. Our proof assumes the technical assumption $d(\mathbf{K}) \geq 4^{N} d(\mathbf{k})^{2}$ where $N$ is the degree of $\mathbf{k}$ and where $d(\mathbf{k})$ and $d(\mathbf{K})$ are the absolute values of the discriminants of $\mathbf{k}$ and $\mathbf{K}$.

Let us stress that, under this previous technical assumption, one remarkable consequence of Theorem 1 is that the zeta function of $\mathbf{k}$ has no real zeros in the open interval ] 0,1 [ provided that the relative class number of $\mathbf{K}$ is less than or equal to 2 (or provided that this relative class number is not "too large"). If we can deduce from this that the zeta function of $\mathbf{K}$ is nonpositive on this interval, then from our previous lower bounds for the relative class numbers we may get very good upper bounds on the discriminants of $\mathbf{K}$, provided that the relative class number of $\mathbf{K}$ is less than or equal to 2 (or provided that this relative class number if not "too large").

Our main application of these techniques is the proof in Corollary $b$ that the zeta function of the real quadratic subfield $\mathbf{k}$ of a totally imaginary cyclic

Received by the editors March 17, 1992 and, in revised form, June 5, 1992.

1991 Mathematics Subject Classification. Primary 11R42, 11R29.

Key words and phrases. Class number, CM-fields, zeta function. 
quartic number field $\mathbf{K}$ with ideal class group of exponent $\leq 2$ and discriminant $d(\mathbf{K})$ has no real zero in the interval $1-2 / \log (d(\mathbf{K})) \leq s<1$. This is the result we needed in [5] in order to prove that there are exactly 33 such quartic number fields.

Let $\mathbf{k}$ be an algebraic number field with class number $h(\mathbf{k})$ and regulator $\operatorname{Reg}(\mathbf{k})$. Let $d(\mathbf{k})$ be the absolute value of the discriminant of $\mathbf{k}$. Set

$$
\begin{gathered}
{[\mathbf{k}: \mathbf{Q}]=r_{1}+2 r_{2}, \quad A=2^{-r_{2}} d(\mathbf{k})^{1 / 2} \pi^{-\left(r_{1}+2 r_{2}\right) / 2},} \\
\lambda(\mathbf{k})=\frac{2^{r_{1}} h(\mathbf{k}) \operatorname{Re} g(\mathbf{k})}{w(\mathbf{k})} \quad \text { where } w(\mathbf{k}) \text { is the number of roots of unity of } \mathbf{k}, \\
F_{\mathbf{k}}(s)=A^{s} \Gamma\left(\frac{s}{2}\right)^{r_{1}} \Gamma(s)^{r_{2}} \zeta_{\mathbf{k}}(s),
\end{gathered}
$$

so that $F_{\mathbf{k}}(s)$ has a simple pole at $s=1$ with residue equal to $\lambda(\mathbf{k})$.

Whenever $y \in\left(\mathbf{R}_{+}^{*}\right)^{r_{1}+r_{2}}$, we set

$$
\begin{gathered}
\|y\|_{\mathbf{k}}=\left(y_{1} \cdots \cdot y_{r_{1}}\right)\left(y_{r_{1}+1} \cdots \cdot y_{r_{1}+r_{2}}\right)^{2} \text {, so that }\|\lambda y\|_{\mathbf{k}}=\lambda^{N}\|y\|, \lambda>0 ; \\
\operatorname{Tr}_{\mathbf{k}}(y)=y_{1}+\cdots+y_{r_{1}}+2\left(y_{r_{1}+1}+\cdots+y_{r_{1}+r_{2}}\right) \text {, so that } \operatorname{Tr}_{\mathbf{k}}(\lambda y)=\lambda y .
\end{gathered}
$$

It is well known (see [4]) that we have the integral representation

$$
F_{\mathbf{k}}(s)=\frac{\lambda(\mathbf{k})}{s(s-1)}+I_{\mathbf{k}}(s)
$$

with

(1)

$$
I_{\mathbf{k}}(s)=\sum_{\mathbf{B}} \int_{\|y\|_{\mathbf{k}} \geq 1} \exp \left(-\pi d(\mathbf{k})^{-1 / N} N_{\mathbf{k} / \mathbf{Q}}(\mathbf{B})^{2 / N} \operatorname{Tr}_{\mathbf{k}}(y)\right)\left[\|y\|_{\mathbf{k}}^{s / 2}+\|y\|_{\mathbf{k}}^{(1-s) i^{2}}\right] \frac{d y}{y},
$$

where the sum is taken over all integral ideals $\mathbf{B} \neq 0$ of $\mathbf{k}$.

From now on, we assume that $s$ is a real number such that $\frac{1}{2} \leq s<1$.

For $y=\left(y_{1}, \ldots, y_{N}\right) \in\left(\mathbf{R}_{+}^{*}\right)^{N}$ we set $\operatorname{Tr}(y)=y_{1}+\cdots+y_{N}$ and $\|y\|=$ $y_{1} \cdots \cdots y_{N}$.

If $\mathbf{K}$ is a totally imaginary number field of degree $2 N$ that is a quadratic extension of a totally real number field $\mathbf{k}$ of degree $N$, then $I_{\mathbf{K}}(s)$ and $I_{\mathbf{k}}(s)$ are integrals in $\left(\mathbf{R}_{+}^{*}\right)^{N}$ and we have:

$\operatorname{Tr}_{\mathbf{K}}(y)=2 \operatorname{Tr}(y)$ and $\|y\|_{\mathbf{K}}=\|y\|^{2}$, so that $\|y\|_{\mathbf{K}} \geq 1$ if and only if $\|y\| \geq 1$;

$\operatorname{Tr}_{\mathbf{k}}(y)=\operatorname{Tr}(y)$ and $\|y\|_{\mathbf{k}}=\|y\|$, so that $\|y\|_{\mathbf{k}} \geq 1$ if and only if $\|y\| \geq 1$.

Moreover, we have the natural injection map $i_{\mathbf{K} / \mathbf{k}}$ from the group of fractional ideals of $\mathbf{k}$ in the group of fractional ideals of $\mathbf{K}$ that satisfies

$$
N_{\mathbf{K} / \mathbf{Q}}\left(i_{\mathbf{K} / \mathbf{k}}(\mathbf{B})\right)^{2 / 2 N}=N_{\mathbf{k} / \mathbf{Q}}(\mathbf{B})^{2 / N}
$$

whenever $\mathbf{B}$ is an integral ideal of $\mathbf{k}$. We thus get

(2) $I_{\mathbf{K}}(s) \geq \sum_{\mathbf{B}} \int_{\|y\| \geq 1} \exp \left(-2 \pi d(\mathbf{K})^{-1 / 2 N} N_{\mathbf{k} / \mathbf{Q}}(\mathbf{B})^{2 / N} \operatorname{Tr}(y)\right)\left[\|y\|^{s}+\|y\|^{1-s}\right] \frac{d y}{y}$ and

$$
I_{\mathbf{k}}(s)=\sum_{\mathbf{B}} \int_{\|y\| \geq 1} \exp \left(-\pi d(\mathbf{k})^{-1 / N} N_{\mathbf{k} / \mathbf{Q}}(\mathbf{B})^{2 / N} \operatorname{Tr}_{\mathbf{k}}(y)\right)\left[\|y\|^{s / 2}+\|y\|^{(1-s) / 2}\right] \frac{d y}{y},
$$


where the sums are taken over all integral ideals $\mathbf{B} \neq 0$ of $\mathbf{k}$.

Hence, if we assume that we have $d(\mathbf{K}) \geq 4^{N} d(\mathbf{k})^{2}$, noticing that we have $\|y\|^{2}>\|y\|$ whenever $\|y\|>1$, then (2) and (3) provide us with

$$
I_{\mathbf{K}}(s)>I_{\mathbf{k}}(s) \text {. }
$$

Moreover, from (2) we have

$$
I_{\mathbf{K}}(s) \geq \sum_{\mathbf{B}} \int_{\|y\| \geq 1} \exp \left(-2 \pi d(\mathbf{K})^{-1 / 2 N} N_{\mathbf{k} / \mathbf{Q}}(\mathbf{B})^{2 / N} \operatorname{Tr}(y)\right)\|y\|^{s} \frac{d y}{y}
$$

and from (3) we have

$$
I_{\mathbf{k}}(s) \leq 2 \sum_{\mathbf{B}} \int_{\|y\| \geq 1} \exp \left(-\pi d(\mathbf{k})^{-1 / N} N_{\mathbf{k} / \mathbf{Q}}(\mathbf{B})^{2 / N} \operatorname{Tr}(y)\right)\|y\|^{s / 2} \frac{d y}{y},
$$

where the sums are taken over all integral ideals $\mathbf{B} \neq 0$ of $\mathbf{k}$.

We change variables in (4), making the multiplicative translation $y=$ $d(\mathbf{K})^{1 / 2 N} Y / 2 d(\mathbf{k})^{1 / N}$. We note that, under the hypothesis $d(\mathbf{K}) \geq 4^{N} d(\mathbf{k})^{2}$, the domain $\|Y\| \geq 1$ is included in the domain $\|Y\| \geq 2^{N} d(\mathbf{k}) / \sqrt{d(\mathbf{K})}$. Using $\|Y\|^{s}>\|Y\|^{s / 2}$ whenever $\|Y\|>1$, we get

$$
\begin{gathered}
I_{\mathbf{K}}\left(\frac{1}{2}\right)>\left(\frac{d(\mathbf{K})}{4^{N} d(\mathbf{k})^{2}}\right)^{1 / 4} I_{\mathbf{k}}\left(\frac{1}{2}\right), \\
I_{\mathbf{K}}(s)>\frac{1}{2}\left(\frac{d(\mathbf{K})}{4^{N} d(\mathbf{k})^{2}}\right)^{s / 2} I_{\mathbf{k}}(s), \quad \frac{1}{2}<s<1 .
\end{gathered}
$$

Now, as $\mathbf{K}$ is a CM-field of degree $2 N$ that is a quadratic extension of a totally real number field $\mathbf{k}$ of degree $N$, it is well known that we have

$$
\frac{\lambda(\mathbf{K})}{\lambda(\mathbf{k})}=\frac{h^{*}(\mathbf{K})}{Q w(\mathbf{K})},
$$

where $h^{*}(\mathbf{K})$ is the relative class number of $\mathbf{K}$ and where $Q=1$ or 2 (see [12, Theorem 4.12]). Moreover, if $\frac{1}{2} \leq s_{0}<1$ is a real zero of $\zeta_{\mathbf{k}}$, then we have $\zeta_{\mathbf{K}}\left(s_{0}\right)=0$ since $\mathbf{K} / \mathbf{k}$ is normal, so that $F_{\mathbf{k}}\left(s_{0}\right)=F_{\mathbf{K}}\left(s_{0}\right)=0$, so that

$$
\frac{\lambda(\mathbf{K})}{\lambda(\mathbf{k})}=\frac{I_{\mathbf{K}}\left(s_{0}\right)}{I_{\mathbf{k}}\left(s_{0}\right)} .
$$

Hence, we get the following theorem whose assertion $(b)$ is much more precise than the one given in [6] (note that as soon as the totally real number field $\mathbf{k}$ is fixed, then there are only finitely many totally imaginary number fields $\mathbf{K}$ that are quadratic extensions of $\mathbf{k}$ and such that $\left.d(\mathbf{K})<4^{N} d(\mathbf{k})^{2}\right)$ :

Theorem 1. Let $\mathbf{K}$ be a CM-field of degree $2 N$ that is a quadratic extension of a totally real number field $\mathbf{k}$ of degree $N$. Let us suppose that we have $d(\mathbf{K}) \geq 4^{N} d(\mathbf{k})^{2}$.

If the Dedekind zeta function of $\mathbf{k}$ has a real zero $s_{0}$ such that $\frac{1}{2} \leq s_{0}<1$, then we have the three following lower bounds for the relative class number $h^{*}(\mathbf{K})$ 
of $\mathbf{K}$ :
(a) $h^{*}(\mathbf{K})>Q w(\mathbf{K}) \geq 2$,
(b) $h^{*}(\mathbf{K})>\frac{Q w(\mathbf{K})}{\sqrt{2^{N} d(\mathbf{k})}} d(\mathbf{K})^{1 / 4}$ if $s_{0}=\frac{1}{2}$,
(c) $h^{*}(\mathbf{K})>\frac{1}{2} Q w(\mathbf{K})\left(\frac{d(\mathbf{K})}{4^{N} d(\mathbf{k})^{2}}\right)^{s_{0} / 2}$ if $\frac{1}{2}<s_{0}<1$.

Hence, the zeta function of $\mathbf{k}$ has no real zero in the interval $1-2 / \log (d(\mathbf{K})) \leq$ $s<1$ provided that we have $h^{*}(\mathbf{K}) \leq \sqrt{d(\mathbf{K})} / e 2^{N} d(\mathbf{k})$.

Remark. Theorem 1 does not apply to the class number one problem for cyclotomic fields, for $d(\mathbf{K})=d(\mathbf{k})^{2}$ whenever $\mathbf{K}=\mathbf{Q}\left(\zeta_{n}\right)$ with $n$ not a prime power, and $d(\mathbf{K})=p d(\mathbf{k})^{2}$ whenever $\mathbf{K}=\mathbf{Q}\left(\zeta_{p^{a}}\right)$, with $p$ an odd prime. Nevertheless, in Corollary $\mathrm{c}$, we will manage to consider the class number one problem for cyclotomic fields (with prime powers conductors). Theorem 1 does not apply to the class number one problem for totally imaginary biquadratic abelian number fields with group $(\mathbf{Z} / 2 \mathbf{Z})^{2}$, for $d(\mathbf{K})=d(\mathbf{k})^{2}$ whenever $\mathbf{K}=\mathbf{Q}(\sqrt{-p}, \sqrt{-q})$, $p$ and $q$ prime and congruent to $3 \bmod 4$.

In fact, Theorem 1 applies nicely to class numbers problems for totally imaginary cyclic number fields with bounded degrees.

Theorem 2. Let $\mathbf{K}$ be a CM-field of degree $2 N$ that is a quadratic extension of a totally real number field $\mathbf{k}$ of degree $N$. Let $\operatorname{Res}_{1}\left(\zeta_{\mathbf{k}}\right)$ be the residue at $s=1$ of the Dedekind zeta function $\zeta_{\mathbf{k}}$ of $\mathbf{k}$. Let us suppose that the Dedekind zeta function $s \mapsto \zeta_{\mathbf{K}}(s)$ satisfies

$$
\zeta_{\mathbf{K}}(1-2 / \log (d(\mathbf{K}))) \leq 0 .
$$

Then, we have the following lower bounds for the relative class number of $\mathbf{K}$ :

$$
h^{*}(\mathbf{K}) \geq f(N, \mathbf{K}) \frac{1}{\operatorname{Res}_{1}\left(\zeta_{\mathbf{k}}\right)} \frac{2 Q w(\mathbf{K})}{e(2 \pi)^{N}} \frac{\sqrt{d(\mathbf{K}) / d(\mathbf{k})}}{\log (d(\mathbf{K}))},
$$

with the two possible choices:

$$
f(N, \mathbf{K})=1-\frac{2 \pi N e^{1 / N}}{d(\mathbf{K})^{1 / 2 N}}
$$

or

$$
f(N, \mathbf{K})=\frac{2}{5} \exp \left(-\frac{2 \pi N}{d(\mathbf{K})^{1 / 2 N}}\right), \quad \text { whenever } N \geq 2 .
$$

Proof. We get the desired result from Proposition A thanks to

$$
\frac{\operatorname{Res}_{1}\left(\zeta_{\mathbf{K}}\right)}{\operatorname{Res}_{1}\left(\zeta_{\mathbf{k}}\right)}=(2 \pi)^{N} \sqrt{\frac{d(\mathbf{k})}{d(\mathbf{K})}} \frac{\lambda(\mathbf{K})}{\lambda(\mathbf{k})}=(2 \pi)^{N} \frac{h^{*}(\mathbf{K})}{Q w(\mathbf{K})} \sqrt{\frac{d(\mathbf{k})}{d(\mathbf{K})}} .
$$

Proposition A. Let $\mathbf{K}$ be a totally imaginary number field of degree $2 N$. If its Dedekind zeta function $s \mapsto \zeta_{\mathbf{K}}(s)$ is such that $\zeta_{\mathbf{K}}\left(s_{0}\right) \leq 0$ for some $s_{0}$ real in $\left[\frac{1}{2}, 1[\right.$, then we have the following effective lower bounds for the residue at $s=1$ of this zeta function:

$$
\operatorname{Res}_{1}\left(\zeta_{\mathbf{K}}\right) \geq\left(1-s_{0}\right) d(\mathbf{K})^{\left(s_{0}-1\right) / 2}\left\{1-\frac{2 \pi N}{d(\mathbf{K})^{s_{0} / 2 N}}\right\}
$$

(b) $\operatorname{Res}_{1}\left(\zeta_{\mathbf{K}}\right) \geq \frac{2}{5}\left(1-s_{0}\right) d(\mathbf{K})^{\left(s_{0}-1\right) / 2} \exp \left(-\frac{2 \pi N}{d(\mathbf{K})^{1 / 2 N}}\right), \quad$ whenever $N \geq 2$. 
Proof. From (1) where we use only the term of the sum corresponding to the ideal $\mathbf{B}$ equal to the ring of algebraic integers of $\mathbf{K}$, and where we disregard the term with $\|y\|_{\mathbf{K}}^{(1-s) / 2}$, we get

$$
\frac{\sqrt{d(\mathbf{K})}}{(2 \pi)^{N}} \frac{\operatorname{Res}_{1}\left(\zeta_{\mathbf{K}}\right)}{s_{0}\left(1-s_{0}\right)}=\frac{\lambda(\mathbf{K})}{s_{0}\left(1-s_{0}\right)} \geq \int_{\|y\| \geq 1} \exp \left(-2 \pi d(\mathbf{K})^{-1 / 2 N} \operatorname{Tr}(y)\right)\|y\|^{s_{0}} \frac{d y}{y} .
$$

Setting $y=d(\mathbf{K})^{1 / 2 N} Y$, we get

$$
\begin{aligned}
\frac{\operatorname{Res}_{1}\left(\zeta_{\mathbf{K}}\right)}{(2 \pi)^{N}} & \geq s_{0}\left(1-s_{0}\right) d(\mathbf{K})^{(s-1) / 2} \int_{\|Y\| \geq d(\mathbf{K})^{-1 / 2}} \exp (-2 \pi \operatorname{Tr}(Y))\|Y\|^{s_{0}} \frac{d Y}{Y} \\
& =\left(1-s_{0}\right) d(\mathbf{K})^{\left(s_{0}-1\right) / 2}\left\{f\left(s_{0}\right)-J_{\mathbf{K}}\left(s_{0}\right)\right\}
\end{aligned}
$$

with

$$
f(s)=s\left[\frac{\Gamma(s)}{(2 \pi)^{s}}\right]^{N} \text { and } J_{\mathbf{K}}(s)=s \int_{\|Y\| \leq d(\mathbf{K})^{-1 / 2}} \exp (-2 \pi \operatorname{Tr}(Y))\|Y\|^{s} \frac{d Y}{Y}
$$

Since $\left\{Y ;\|Y\| \leq d(\mathbf{K})^{-1 / 2}\right\}$ is included in $\left\{Y ; \exists i \in\{1, \ldots, N\} / Y_{i} \leq\right.$ $\left.d(\mathbf{K})^{-1 / 2 N}\right\}$, we have (using $e^{-2 \pi y} \leq 1, y \geq 0$ )

$$
J_{\mathbf{K}}(s) \leq N s\left[\frac{\Gamma(s)}{(2 \pi)^{s}}\right]^{N-1} \int_{0}^{d(\mathbf{K})^{-1 / 2 N}} e^{-2 \pi y} y^{s} \frac{d y}{y} \leq N f(s) \frac{(2 \pi)^{s}}{s \Gamma(s)} d(\mathbf{k})^{-s / 2 N} .
$$

Hence,

$$
\frac{\operatorname{Res}_{1}\left(\zeta_{\mathbf{K}}\right)}{(2 \pi)^{N}} \geq\left(1-s_{0}\right) d(\mathbf{K})^{\left(s_{0}-1\right) / 2} f\left(s_{0}\right)\left\{1-N \frac{(2 \pi)^{s_{0}}}{s_{0} \Gamma\left(s_{0}\right)} d(\mathbf{K})^{-s_{0} / 2 N}\right\} .
$$

Since $s \mapsto f(s)$ decreases on $] 0,1\left[\right.$, we have $f\left(s_{0}\right) \geq f(1)=(1 / 2 \pi)^{N}$. Since $s \mapsto(2 \pi)^{s} / s \Gamma(s)$ increases on ]0, 1[, we get the desired first result. In order to get the second desired result, we start from (6) and use the third point of the following lemma with $x=d(\mathbf{K})^{-1 / 2 N}$ (so that from the Minkowski's lower bound $d(\mathbf{K})^{1 / 2 N} \geq \pi N^{2} /((2 N) !)^{1 / N}$ we have $\left.x \leq \frac{1}{2}, 2 N \geq 4\right)$ :

Lemma. Set $P_{N}(t)=\sum_{n=0}^{N-1} t^{n} / n$ !. Then,

(i)

(ii)

$$
\int_{\operatorname{Tr}(Y) \geq t} \exp (-\operatorname{Tr}(Y)) d Y=P_{N}(t) e^{-t}, \quad N \geq 1
$$

$$
\int_{\operatorname{Tr}(Y) \leq t} \exp (-\operatorname{Tr}(Y)) d Y=1-P_{N}(t) e^{-t}, \quad N \geq 1
$$

$$
\int_{1 \geq\|y\| \geq x^{N}} \exp (-2 \pi \operatorname{Tr}(y)) d y=\frac{e^{-2 \pi N x}}{(2 \pi)^{N}}\left(1-P_{N}(2 \pi N(1-x)) e^{-2 \pi N(1-x)}\right) .
$$

(iv) $x_{N}=1-P_{N}(\pi N) e^{-\pi N}$ is an increasing sequence that converges towards 1 , so that $x_{N} \geq \frac{4}{5}, N \geq 2$.

Proof. Part (iii) is proved from (ii) using the fact that the domain $\{y ; y \in$ $\left(\mathbf{R}_{+}^{*}\right)^{N}, y_{i} \geq x$ and $\left.N \geq \operatorname{Tr}(y)\right\}$ is included in the domain $\left\{y ; y \in\left(\mathbf{R}_{+}^{*}\right)^{N}\right.$ and 
$\left.1 \geq\|y\| \geq x^{N}\right\}$ and changing variables making the translation $y_{i}=x+Y_{i} / 2 \pi$. Part (iv) follows from the inequality $P_{N+1}((N+1) \pi) \leq e^{\pi} P_{N}(N \pi)$. Indeed, we have

$$
\begin{aligned}
P_{N+1}((N+1) \pi) & =\sum_{n=0}^{N} \frac{(\pi N)^{n}}{n !}\left(\frac{N+1}{N}\right)^{n} \leq\left(\frac{N+1}{N}\right)^{N} \sum_{n=0}^{N} \frac{(\pi N)^{n}}{n !} \\
& \leq e\left(P_{N}(N \pi)+\frac{(N \pi)^{N}}{N !}\right)=e\left(P_{N}(N \pi)+\pi \frac{(N \pi)^{N-1}}{(N-1) !}\right) \\
& \leq e(1+\pi) P_{N}(N \pi) .
\end{aligned}
$$

Remark. Theorems 1 and 2 apply nicely to the determination of CM-fields $\mathbf{K}$ with "small" class numbers, provided that the fields $\mathbf{K}$ are CM-fields that are quadratic extensions of totally real number fields $\mathbf{k}$ such that $\zeta_{\mathbf{K}} / \zeta_{\mathbf{k}}$ is nonnegative on ]0,1[. Indeed, Theorem 1 then implies that the zeta functions $\zeta_{\mathbf{k}}$ have no real zero in the interval $1-2 / \log (d(\mathbf{K})) \leq s<1$. Hence, $\zeta_{\mathbf{k}}\left(s_{0}\right)<0$ and $\zeta_{\mathbf{K}}\left(s_{0}\right) \leq 0$ with $s_{0}=1-2 / \log (d(\mathbf{K}))$, so that Theorem 2 provides us with good lower bounds for $h^{*}(\mathbf{K})$. Since we seek "small" class numbers, this will provide us with upper bounds on $d(\mathbf{K})$.

Let us point out that these assumptions " $\zeta_{\mathbf{K}} / \zeta_{\mathbf{k}}$ are nonnegative on $] 0,1[$ " are satisfied as soon as the number fields $\mathbf{K}$ are totally imaginary and cyclic over $\mathbf{Q}$ and such that 4 divides $[\mathbf{K}: \mathbf{Q}]=2 N$, for $\zeta_{\mathbf{K}} / \zeta_{\mathbf{k}}$ is then a product of $L$-functions that come in conjugate pairs.

For example, we first give the following corollary, which greatly improves upon the upper bounds given in [1] or [10]:

Corollary $\mathbf{a}$. Let $\mathbf{K}$ be a cyclic quartic totally imaginary number field with conductor $f$ and class number $h(\mathbf{K})$. If $h(\mathbf{K})=1$ then $f \leq 4500$. If $h(\mathbf{K})=2$ then $f \leq 10000$.

Let $\mathbf{K}$ be a cyclic octic totally imaginary number field with conductor $f$ and class number $h(\mathbf{K})$. If $h(\mathbf{K})=1$ then $f=32$ or $f$ is prime and $f \leq 3000$.

Proof. We only prove the first point. Let $\mathbf{k}$ be the real quadratic subfield of $\mathbf{K}$, let $f_{\mathbf{k}}$ be the conductor of $\mathbf{k}$, and let $L\left(s, \chi_{f_{\mathbf{k}}}\right)$ be the $L$-function of $\mathbf{k}$. First, $f_{\mathbf{k}}$ divides $f$, so that we have $f_{\mathbf{k}} \leq f$. Moreover, $d(\mathbf{k})=f_{\mathbf{k}}$ and $d(\mathbf{K})=f_{\mathbf{k}} f^{2}$. Hence, $d(\mathbf{K}) / 4^{2} d(\mathbf{k})^{2}=f^{2} / 16 f_{\mathbf{k}}$ is greater than or equal to 1 as soon as we have $f \geq 16$. Hence, from Theorem 1(a) we deduce that the Dedekind zeta function of $\mathbf{k}$ has no zero on the interval $\left[\frac{1}{2}, 1\right.$ [ as soon as $h^{*}(\mathbf{K})=1$ or 2 , provided that we have $f \geq 16$. Since the Dedekind zeta function of $\mathbf{K}$ can be written $\left.\zeta_{\mathbf{K}}(s)=\zeta_{\mathbf{k}}(s)\left|L\left(s, \chi_{f}\right)\right|^{2}, s \in\right] 0,1[$, we can apply Theorem 2 . Since $\operatorname{Res}_{1}\left(\zeta_{\mathbf{k}}\right)=L\left(1, \chi_{f_{\mathbf{k}}}\right) \leq \frac{1}{2} \log \left(f_{\mathbf{k}}\right)+1 \leq \frac{1}{2} \log (f)+1$ (see [8, Lemma 8.4]) and since $5 f^{2} \leq d(\mathbf{K}) \leq f^{3}$, Theorem 2 provides us with the following lower bound from which we get the desired results:

$$
h(\mathbf{K}) \geq h^{*}(\mathbf{K}) \geq \frac{2}{3 e \pi^{2}}\left(1-\frac{4 \pi e^{1 / 2}}{\left(5 f^{2}\right)^{1 / 4}}\right) \frac{f}{(\log (f)+2) \log (f)} .
$$

Corollary $\mathbf{b}$. Let $\mathbf{K}$ be a cyclic quartic totally imaginary number field with conductor $f$. Then the Dedekind zeta function of the real quadratic subfield $\mathbf{k}$ of $\mathbf{K}$ has no zero in the interval $[1-2 / \log (d(\mathbf{K})), 1[$ provided that the ideal class group of $\mathbf{K}$ is of exponent $\leq 2$. 
Proof. If the ideal class group of $\mathbf{K}$ is of exponent $\leq 2$, then $\mathbf{k}$ is principal and $f_{\mathbf{k}}=8$ or $f_{\mathbf{k}}$ is prime and such that $f_{\mathbf{k}} \equiv 1(\bmod 4)$. Conversely, if $f_{\mathbf{k}} \equiv 1$ $(\bmod 4)$ is prime, if $\mathbf{k}$ is principal, and if we define $f_{2}$ by means of $f=f_{\mathbf{k}} f_{2}$, then the ideal class group of $\mathbf{K}$ has 2-rank $t-1$ where $t$ is the number of prime ideals that ramify in the quadratic extension $\mathbf{K} / \mathbf{k}$. Hence, $t \leq 1+2 \omega\left(f_{2}\right)$ where $\omega\left(f_{2}\right)$ is the number of prime divisors of $f_{2}$ (the proofs of these assertions can be found in [5]). Let us suppose that the Dedekind zeta function of $\mathbf{k}$ had a real zero $s_{0}$ such that $1-2 / \log (d(\mathbf{K})) \leq s_{0}<1$. Then, as $d(\mathbf{K})=f_{\mathbf{k}} f^{2}=f_{\mathbf{k}}^{3} f_{2}^{2}$ and $d(\mathbf{k})=f_{\mathbf{k}}$, Theorem 1 would imply $4^{\omega\left(f_{2}\right)} \geq h(\mathbf{K}) \geq h^{*}(\mathbf{K}) \geq \sqrt{f_{\mathbf{k}}} f_{2} / 4 e$.

Now, $f_{\mathbf{k}} \geq 211$ implies $\sqrt{f_{k}} / 4 e>4 / 3$, so that we would have $4^{\omega\left(f_{2}\right)}>$ $4 f_{2} / 3$. Since 4 divides $f_{2}$ as soon as $f_{2}$ is even, this inequality is never satisfied. On the other hand, if $5 \leq f_{\mathbf{k}} \leq 211$, then $s \mapsto L\left(s, \chi_{f_{\mathbf{k}}}\right)$ has no zero on ]0, 1[ (see [9]). Thus, we get the desired result.

Lower bounds for the relative class numbers of cyclotomic fields. Now, we would like to show that Theorem 2 applies to CM-fields with unbounded degrees. For example, we show that Theorem 2(b) enables us to get good upper bounds on the conductors of the cyclotomic fields (with prime-power conductors) with relative class numbers equal to 1 . We first give a less tedious proof and more precise form of Lemma 11.5 of Washington [12]; i.e., we give an upper bound on $\operatorname{Res}_{1}(\mathbf{k})$ with $\mathbf{k}$ being the maximal totally real subfield of a cyclotomic field with prime power conductor.

We define $g(b)=b-1+H(b, 1)$, where

$$
H(b, s)=\sum_{n \geq 0}\left(\frac{1}{(n+b)^{s}}-\frac{1}{(n+1)^{s}}\right), \quad\left\{\begin{array}{l}
\operatorname{Re}(s)>0 \\
b>0
\end{array}\right.
$$

Whenever $\chi: \mathbf{N} \mapsto \mathbf{C}$ is a complex-valued function which is periodic mod $m$, such that $\chi(m)=0$ and $\sum_{a=1}^{m-1} \chi(a)=0$, we have

$$
L(s, \chi)=\sum_{n \geq 1} \frac{\chi(n)}{n^{s}}=\frac{1}{m^{s}} \sum_{a=1}^{m-1} \chi(a) H\left(\frac{a}{m}, s\right) .
$$

Consequently, whenever $\chi_{m}$ is a nontrivial (not necessarily primitive) even Dirichlet character mod $m$ we have $\sum_{a=1}^{m-1} a \chi_{m}(a)=0$ and

$$
L\left(1, \chi_{m}\right)=\frac{1}{m} \sum_{a=1}^{m-1} \chi(a) g\left(\frac{a}{m}\right) \text {. }
$$

Lemma (i). $g(b) \geq 0$ and $g(b)^{2}+g(b) g(1-b) \leq 1 / b^{2}, 0<b<1$.

Proof. Follows from the following two inequalities:

$$
\begin{aligned}
& g(b)=b-1+\frac{1}{b}-1+\sum_{n \geq 1}\left(\frac{1}{n+b}-\frac{1}{n+1}\right) \geq b+\frac{1}{b}-2=\frac{(1-b)^{2}}{b} \geq 0 \\
& g(b)=b-1+\frac{1}{b}-\sum_{n \geq 1} \frac{b}{n(n+b)} \leq b-1+\frac{1}{b}-\sum_{n \geq 1} \frac{b}{n(n+1)}=\frac{1}{b}-1 .
\end{aligned}
$$

Lemma (ii). $\left|\prod_{\chi_{m} \text { even, } \chi_{m} \neq 1} L\left(1, \chi_{m}\right)\right| \leq\left(\pi^{2} / 6\right)^{(\phi(m)-2) / 4}$, where the product is over the (not necessarily primitive) even Dirichlet characters $\bmod m$. 
Proof.

$$
\sum_{\substack{\chi_{m} \text { even } \\ \chi_{m} \neq 1}} \chi_{m}(a) \bar{\chi}_{m}(b)= \begin{cases}\phi(m) / 2-1 & \text { if } a \equiv \pm b(\bmod m) \\ & \text { and } \operatorname{GCD}(a, m)=1, \\ 0 \text { or }-1 & \text { otherwise. }\end{cases}
$$

Hence, by the arithmetic-geometric mean inequality,

$$
\begin{aligned}
& \left(\prod_{\substack{\chi_{m} \text { even } \\
\chi_{m} \neq 1}}\left|L\left(1, \chi_{m}\right)\right|^{2}\right)^{2 /(\phi(m)-2)} \\
& \leq \frac{2 m^{-2}}{\phi(m)-2} \sum_{a=1}^{m-1} \sum_{b=1}^{m-1} g\left(\frac{a}{m}\right) g\left(\frac{b}{m}\right)\left(\sum_{\substack{\chi_{m} \text { even } \\
\chi_{m} \neq 1}} \chi_{m}(a) \bar{\chi}_{m}(b)\right) \\
& \leq \frac{1}{m^{2}} \sum_{\substack{a=1 \\
m-1}}^{m} g\left(\frac{a}{m}\right)^{2}+g\left(\frac{a}{m}\right) g\left(1-\frac{a}{m}\right) \leq \sum_{\substack{a=1 \\
(a, m)=1}}^{m-1} \frac{1}{a^{2}} \\
& \leq \sum_{\substack{a=1 \\
(a, m)=1}}^{+\infty} \frac{1}{a^{2}}=\frac{\pi^{2}}{6} \prod_{\substack{p \text { prime } \\
p \text { divides } m}}\left(1-\frac{1}{p^{2}}\right) \leq \frac{\pi^{2}}{6} . \square
\end{aligned}
$$

Now, in order to apply Theorem 2 to the cyclotomic case, and thanks to the fact that the Dedekind zeta function of a CM cyclic number field factorises on ]0, 1 [ into a product of $L$-functions that come in conjugate pairs, apart from the two $L$-functions associated to the principal character and to some quadratic character, we must find an explicit zero-free region for an $L$-function associated to a quadratic character.

Lemma (iii). Let $\chi$ be a quadratic nonprincipal character $\bmod f$. Set $N=$ $\frac{1}{2} \varphi(f)$. Then, for $\sigma \geq 1 / \log (3)$ we have

$$
\left|L^{\prime}(\sigma, \chi)\right| \leq \sum_{n=2}^{N+2} \frac{\log (n)}{n^{\sigma}} \leq(N+2)^{1-\sigma} \sum_{n=2}^{N+2} \frac{\log (n)}{n} .
$$

Proof. We have

$$
L^{\prime}(\sigma, \chi)=-\chi(2) \frac{\log (2)}{2^{\sigma}}-\sum_{k \geq 0}\left(\sum_{n=k f+3}^{(k+1) f+2} \chi(n) \frac{\log (n)}{n^{\sigma}}\right) .
$$

Now, $n \mapsto \log (n) / n^{\sigma}$ decreases for $n \geq 3$ provided that we have $\sigma \geq$ $1 / \log (3)$. Moreover, in each set of $f$ consecutive integers there are $N$ of them such that $\chi(n)=-1$ and $N$ of them such that $\chi(n)=+1$. Hence, for each $k \geq 0$ we have

$$
\left|\sum_{n=k f+3}^{(k+1) f+2} \chi(n) \frac{\log (n)}{n^{\sigma}}\right| \leq u_{k}-v_{k}
$$


with

$$
u_{k}=\sum_{n=k f+3}^{k f+N+2} \frac{\log (n)}{n^{\sigma}} \text { and } v_{k}=\sum_{n=(k+1) f+3-N}^{(k+1) f+2} \frac{\log (n)}{n^{\sigma}} .
$$

Since $\left(u_{k}\right)_{k \geq 0}$ and $\left(v_{k}\right)_{k \geq 0}$ are decreasing sequences converging towards 0 , and since $u_{k+1} \leq v_{k}, k \geq 0$, we get

$$
\begin{aligned}
\left|L^{\prime}(\sigma, \chi)\right| & \leq \frac{\log (2)}{2^{\sigma}}+u_{0}-\left(v_{0}-u_{1}\right)-\left(v_{1}-u_{2}\right)+\cdots \\
& \leq \frac{\log (2)}{2^{\sigma}}+u_{0}=\sum_{n=2}^{N+2} \frac{\log (n)}{n^{\sigma}} \cdot \square
\end{aligned}
$$

Theorem 3 (see [12, Lemma 11.10]). Let $\chi$ be a primitive quadratic character of conductor $f$. Then

$$
L(\sigma, \chi) \geq 0 \text { for } \begin{cases}\sigma \geq \sigma_{0}=1-\frac{2}{\sqrt{f} \log (f)} & \text { if } \chi(-1)=+1 \\ \sigma \geq \sigma_{0}=1-\frac{2 \pi}{\sqrt{f} \log ^{2}(f)} & \text { if } \chi(-1)=-1\end{cases}
$$

Hence, $L(\sigma, \chi) \geq 0$ for $\sigma \geq \sigma_{1}=1-2 /(f-2) \log (f)$.

Proof. Since $\sigma \mapsto L(\sigma, \chi)$ has no real zero in the open interval ]0, 1[ for $f \leq 24$ (see [9]), we may assume that we have $f \geq 24$. Let us first assume that $\chi$ is even, and let $\mathbf{k}_{2}$ be the real quadratic field with conductor $f$. Then $L(1, \chi)=2 h \log \left(\varepsilon_{0}\right) / \sqrt{f} \geq \log (f-4) / \sqrt{f}$ where $\varepsilon_{0} \geq(\sqrt{f-4}+\sqrt{f}) / 2 \geq$ $\sqrt{f-4}$ is the fundamental unit of $\mathbf{k}_{2}$ and where $h \geq 1$ is the class number of $\mathbf{k}_{2}$. Let $\sigma$ be such that $\sigma_{0} \leq \sigma \leq 1$. Then $L(\sigma, \chi) \geq 0$. Indeed, if we had $L(\sigma, \chi)<0$, then from Lemma (iii) above and since we have $N \leq(f-1) / 2$, we would get a contradiction from

$$
\begin{aligned}
\frac{\log (f-4)}{\sqrt{f}} & \leq L(1, \chi)<L(1, \chi)-L(\sigma, \chi) \\
& \leq(1-\sigma) \max _{\sigma_{0} \leq \sigma \leq 1} L^{\prime}(\sigma, \chi) \\
& \leq\left(1-\sigma_{0}\right) \exp \left(\frac{2 \log ((f+3) / 2)}{\sqrt{f} \log (f)}\right) \sum_{2 \leq n \leq(f+3) / 2} \frac{\log (n)}{n} \\
& \leq\left(1-\sigma_{0}\right) \frac{1}{2} \log (f-4) \log (f)=\frac{\log (f-4)}{\sqrt{f}}
\end{aligned}
$$

where the last inequality is valid for $f \geq 24$.

In the same way, we get the desired result if $\chi$ is odd using $L(1, \chi) \geq \pi / \sqrt{f}$, $f \geq 5$.

The third result follows from the first and second ones.

Corollary c (see [12, Corollary 11.17]). Let $p$ be an odd prime. Then we have the following lower bound for the relative class number $h^{*}(\mathbf{K})$ of the cyclotomic field $\mathbf{K}=\mathbf{Q}\left(\zeta_{p^{a}}\right), a \geq 1$, of degree $2 N=[\mathbf{K}: \mathbf{Q}]=\phi\left(p^{a}\right)$ :

$$
h^{*}(\mathbf{K}) \geq \frac{1}{76}\left(\frac{N}{39}\right)^{N / 2} \frac{1}{\log (2 N)},
$$


so that $2 N=\phi\left(p^{a}\right) \geq 100$ implies $h^{*}(\mathbf{K})>1$. Moreover, $p \geq 89$ implies $h^{*}(\mathbf{K})>1$.

Proof. Set $h(p)=(1-1 / p) p^{1 /(p-1)}$, so that we have $h(5) \geq h(p) \geq 1$. We note that

$$
\begin{gathered}
d(\mathbf{K})=(2 N / h(p))^{2 N} \leq(2 N)^{2 N}, \quad w(\mathbf{K})=2 p^{a} \geq 2 N, \\
d(\mathbf{K}) / d(\mathbf{k})=\sqrt{p d(\mathbf{K})}=\sqrt{p}(2 N / h(p))^{N}, \\
\operatorname{Res}_{1}\left(\zeta_{\mathbf{k}}\right) \leq\left(\pi^{2} / 6\right)^{(N-1) / 2} \quad \text { [thanks to Lemma (ii)]. }
\end{gathered}
$$

Noticing that we have $d(\mathbf{K}) \geq p^{p-2}$, then thanks to Theorem 3 we may apply Theorem 2(b), so that we get the following lower bound from which we get the desired results:

$$
h^{*}(\mathbf{K}) \geq \frac{2 \pi \sqrt{6}}{15 e} p^{1 / 4} e^{-\pi h(p)}\left(\frac{3 N}{\pi^{4} h(p)}\right)^{N / 2} \frac{1}{\log (2 N)} .
$$

\section{REFERENCES}

1. K. Hardy, R. H. Hudson, D. Richman, and K. S. Williams, Determination of all imaginary cyclic quartic fields with class number 2, Trans. Amer. Math. Soc. 311 (1989), 1-55.

2. J. Hoffstein, Some analytic bounds for zeta functions and class numbers, Invent. Math. $\mathbf{5 5}$ (1979), 37-47.

3. K. Horie and M. Horie, On the exponent of the class group of CM-fields, Lecture Notes in Math., vol. 1434, Springer-Verlag, Berlin and New York, 1990, pp. 143-148.

4. S. Lang, Functional equation of the zeta function, Hecke's proof, Algebraic Number Theory, Graduate Texts in Math., vol. 110, Springer-Verlag, New York.

5. S. Louboutin, Détermination des corps quartiques cycliques totalement imaginaires à groupes des classes d'idéaux d'exposant $\leq 2$, C. R. Acad. Sci. Paris. Sér. I Math. 315 (1992), 251-254; Manuscripta Math. 77 (1992), 385-404.

6. A. Mallik, A note on Friedlander's paper "On the class numbers of certain quadratic extensions", Acta Arith. 35 (1976), 53-54.

7. M. Masley and H. L. Montgomery, Cyclotomic fields with unique factorization, J. Reine Angew. Math. 286/287 (1976), 248-256.

8. W. Narkiewicz, Elementary and analytic theory of algebraic numbers, PWN, Warsaw, 1976.

9. B. Rosser, Real roots of real Dirichlet series, J. Res. Nat. Bur. Standards 45 (1950), 505-514.

10. H. M. Stark, Some effective cases of the Brauer-Siegel theorem, Invent. Math. 23 (1974), $135-152$.

11. K. Uchida, Imaginary abelian number fields of degree $2^{m}$ with class number one, Proc. Internat. Conf. on Class Numbers and Fundamental Units of Algebraic Number Fields, Kataka, Japan, 1986, pp. 151-170.

12. L. C. Washington, Cyclotomic fields of class number one, Introduction to cyclotomic fields, Graduate Texts in Math., vol. 83, Springer-Verlag, New York, 1982.

Département de Mathématiques, Université de Caen, U. F. R. Sciences, Esplanade de la Paix, 14032 Caen Cedex, France

E-mail address: loubouti@univ-caen.fr 